\title{
A note on the paper "A new general eighth-order family of iterative methods for solving nonlinear equations"
}

\author{
Shima Taher-Khani
}

Received: 16 November 2013 / Accepted: 7 May 2014/Published online: 5 June 2014

(C) The Author(s) 2014. This article is published with open access at Springerlink.com

\begin{abstract}
In this work, we briefly talk about the incorrect convergence order presented in the paper Khan et al. (Appl. Math. Lett. 25:2262-2266, 2012). Accordingly, we first show that their convergence theorem includes major errors, and it is attempted to provide the correct error equation of their method having fifth-order not eighth-order convergence. Finally, to support our assertion, some numerical examples are tested.
\end{abstract}

Keywords Nonlinear equations - Iterative methods · Multipoint methods - Convergence order

\section{Convergence analysis}

Khan et al. [1], proposed the following three-step iterative method

$$
\left\{\begin{array}{l}
y_{m}=x_{m}-\frac{f\left(x_{m}\right)}{f^{\prime}\left(x_{m}\right)}, \\
z_{m}=y_{m}-G\left(\frac{f\left(y_{m}\right)}{f\left(x_{m}\right)}\right) \frac{f\left(y_{m}\right)}{f^{\prime}\left(x_{m}\right)}, \\
x_{m+1}=z_{m}-\frac{\mu}{\lambda+v q_{m}^{2}} \frac{f\left(z_{m}\right)}{K-C\left(y_{m}-z_{m}\right)-D\left(y_{m}-z_{m}\right)^{2}},
\end{array}\right.
$$

where

This research was supported by Islamic Azad University, Takestan Branch.

S. Taher-Khani $(\bowtie)$

Department of Applied Mathematics, Takestan Branch,

Islamic Azad University, Takestan, Iran

e-mail: taherkhani.shima@yahoo.com

$$
\left\{\begin{array}{l}
H=\frac{f\left(x_{m}\right)-f\left(y_{m}\right)}{x_{m}-y_{m}}, \\
K=\frac{f\left(y_{m}\right)-f\left(z_{m}\right)}{y_{m}-z_{m}}, \\
D=\frac{f^{\prime}\left(x_{m}\right)-H}{\left(x_{m}-y_{m}\right)\left(x_{m}-z_{m}\right)}-\frac{H-K}{\left(x_{m}-z_{m}\right)^{2}}, \\
C=\frac{H-K}{\left(x_{m}-y_{m}\right)\left(x_{m}-z_{m}\right)}-D\left(x_{m}+y_{m}-2 z_{m}\right), \\
q_{m}=\frac{f\left(z_{m}\right)}{f\left(x_{m}\right)},
\end{array}\right.
$$

and $\lambda, \mu, v \in R$ and $G(t)$ represent a real-value function.

Khan et al. assert that if the conditions of the following theorem hold, then the iterative method (1.1) has convergence order eight (see Theorem in Section 4 in [1]). However, we prove that this is not true and prove that its convergence order is five.

Theorem 1.0.1 Let $f: D \subset R \rightarrow R$ have a single root $x^{*} \in D$, for an open interval $D$. If the initial point $x_{0}$ is sufficiently close to $x^{*}$, then the sequence $\left\{x_{m}\right\}$ generated by any method of the family (1.1) converges to $x^{*}$. If $G$ is any function with $M_{0}=G(0)=1, M_{1}=G^{\prime}(0)=2, M_{2}=$ $G^{\prime \prime}(0)<\infty$ and $\lambda=\mu \neq 0$, then the methods defined by (1.1) have convergence order of at least 5 .

Proof For the sake of simplicity, we drop the iterative index $m$. Let $e=x-x^{*}$, and $c_{k}=\frac{f^{(k)}\left(x^{*}\right)}{k ! f^{\prime}\left(x^{*}\right)}, k=0,1,2, \ldots$ Since $f\left(x^{*}\right)=0 \neq f^{\prime}\left(x^{*}\right)$, we can write

$$
\begin{aligned}
& f(x)=f^{\prime}\left(x^{*}\right) \sum_{k=1}^{8} c_{k} e^{k}+O\left(e^{9}\right) \\
& f^{\prime}(x)=f^{\prime}\left(x^{*}\right) \sum_{k=1}^{7} k c_{k} e^{k-1}+O\left(e^{8}\right) .
\end{aligned}
$$


Let $e_{y}=y-x^{*}$. Then,

$$
\begin{aligned}
e_{y}= & e-\frac{f(x)}{f^{\prime}(x)} \\
= & c_{2} e^{2}+\left(2 c_{3}-2 c_{2}^{2}\right) e^{3}+\left(4 c_{2}^{3}-7 c_{3} c_{2}+3 c_{4}\right) e^{4} \\
& +\left(-8 c_{2}^{4}+20 c_{3} c_{2}^{2}-10 c_{4} c_{2}-6 c_{3}^{2}\right) e^{5}+O\left(e^{6}\right)
\end{aligned}
$$

Set,

$$
t=\frac{f(y)}{f(x)}=c_{2} e+\left(2 c_{3}-3 c_{2}^{2}\right) e^{2}+\left(8 c_{2}^{3}-10 c_{3} c_{2}+3 c_{4}\right) e^{3}
$$

$$
\begin{aligned}
& +\left(-20 c_{2}^{4}+37 c_{3} c_{2}^{2}-14 c_{4} c_{2}-8 c_{3}^{2}\right) e^{4} \\
& +\left(48 c_{2}^{5}-118 c_{3} c_{2}^{3}+51 c_{4} c_{2}^{2}+55 c_{3}^{2} c_{2}-22 c_{3} c_{4}\right) e^{5} \\
& +O\left(e^{6}\right)
\end{aligned}
$$

Now, define the weight function $G(t)$ by

$$
G(t)=M_{0}+M_{1} t+M_{2} t 2 .
$$

Then,

$$
\begin{aligned}
e_{z}= & z-x^{*}=e_{y}-G(t) \frac{f(y)}{f^{\prime}(x)}-\left(c_{2}\left(M_{0}-1\right)\right) e^{2} \\
& +\left(c_{2}^{2}\left(4 M_{0}-M_{1}-2\right)-2 c_{3}\left(M_{0}-1\right)\right) e^{3} \\
& +\left(c_{2}^{3}\left(-13 M_{0}+7 M_{1}-\frac{M_{2}}{2}+4\right)\right. \\
& \left.+c_{3} c_{2}\left(14 M_{0}-4 M_{1}-7\right)-3 c_{4}\left(M_{0}-1\right)\right) e^{4} \\
& +\left(c_{3} c_{2}^{2}\left(-64 M_{0}+38 M_{1}-3 M_{2}+20\right)\right. \\
& +2 c_{4} c_{2}\left(10 M_{0}-3 M_{1}-5\right)+2 c_{3}^{2}\left(6 M_{0}-2 M_{1}-3\right) \\
& \left.+c_{2}^{4}\left(38 M_{0}-33 M_{1}+5 M_{2}-8\right)\right) e^{5}+O\left(e^{6}\right)
\end{aligned}
$$

If $M_{0}=1$ and $M_{1}=2$, then

$$
\begin{aligned}
e_{z}= & \left(c_{2}^{3}\left(5-\frac{M_{2}}{2}\right)-c_{2} c_{3}\right) e^{4}+\left(c_{2}^{4}\left(5 M_{2}-36\right)\right. \\
& \left.+c_{3} c_{2}^{2}\left(32-3 M_{2}\right)-2 c_{4} c_{2}-2 c_{3}^{2}\right) e^{5}+O\left(e^{6}\right) .
\end{aligned}
$$

Now, assume that $H, K D$, and $C$ are given by (1.2). Also, let $q=\frac{f(z)}{f(x)}$ and $\lambda=\mu \neq 0$. Consequently, the final error equation, i.e., $\hat{e}$, for the method (1.1) is obtained as follows

$$
\begin{aligned}
\hat{e} & =e_{z}-\frac{\mu}{\lambda+v q^{2}} \frac{f(z)}{K-C(y-z)-D(y-z)^{2}} \\
& =\frac{1}{2}\left(c_{2}^{5}\left(M_{2}-10\right)+2 c_{3} c_{2}^{3}\right) e^{5}+O\left(e^{6}\right),
\end{aligned}
$$

which completes the proof.
Table 1 Numerical results for $v=0=\omega$

\begin{tabular}{lllll}
\hline Functions & $\left|x_{1}-x^{*}\right|$ & $\left|x_{2}-x^{*}\right|$ & $\left|x_{3}-x^{*}\right|$ & COC \\
\hline$f_{1}(x)$ & $0.2606(-5)$ & $0.1547(-31)$ & $0.1141(-162)$ & 5.00 \\
$f_{2}(x)$ & $0.60034(-3)$ & $0.3359(-14)$ & $0.1855(-70)$ & 4.99 \\
$f_{3}(x)$ & $0.4018(-2)$ & $0.2348(-12)$ & $0.1509(-63)$ & 5.00 \\
\hline
\end{tabular}

\section{Numerical performances}

This section concerns with numerical results of the proposed methods (1.1). We take the derived method from it by considering $\lambda=\mu=1$ and $G(t)=\frac{1}{1-2 t+\omega t^{2}}$, where $\omega \in R$. This is the Method 1 in [1] (see Equations 21 and 22 in Section 5 there.)

$$
\left\{\begin{array}{l}
y_{m}=x_{m}-\frac{f\left(x_{m}\right)}{f^{\prime}\left(x_{m}\right)}, \\
z_{m}=y_{m}-\frac{f^{2}\left(x_{m}\right)}{f^{2}\left(x_{m}\right)-2 f\left(x_{m}\right) f\left(y_{m}\right)+\omega f^{2}\left(y_{m}\right)} \frac{f\left(y_{m}\right)}{f^{\prime}\left(x_{m}\right)}, \\
x_{m+1}=z_{m}-\frac{1}{1+v q_{m}^{2}} \frac{f\left(z_{m}\right)}{K-C\left(y_{m}-z_{m}\right)-D\left(y_{m}-z_{m}\right)^{2}},
\end{array}\right.
$$

where

$$
\left\{\begin{array}{l}
H=\frac{f\left(x_{m}\right)-f\left(y_{m}\right)}{x_{m}-y_{m}}, \\
K=\frac{f\left(y_{m}\right)-f\left(z_{m}\right)}{y_{m}-z_{m}}, \\
D=\frac{f^{\prime}\left(x_{m}\right)-H}{\left(x_{m}-y_{m}\right)\left(x_{m}-z_{m}\right)}-\frac{H-K}{\left(x_{m}-z_{m}\right)^{2}}, \\
C=\frac{H-K}{\left(x_{m}-y_{m}\right)\left(x_{m}-z_{m}\right)}-D\left(x_{m}+y_{m}-2 z_{m}\right), \\
q_{m}=\frac{f\left(z_{m}\right)}{f\left(x_{m}\right)} .
\end{array}\right.
$$

Numerical results have been carried out using Mathematica 9 with 200 digits of precision. $a(-b)$ means $a \times 10^{b}$. In each table, COC stands for computational order of convergence (see [1]) which is given by

$$
\text { rho } \approx \frac{\ln \left(\left|x_{m+1}-x_{m}\right|\left|x_{m}-x_{m-1}\right|^{-1}\right)}{\ln \left(\left|x_{m}-x_{m-1}\right|\left|x_{m-1}-x_{m-2}\right|^{-1}\right)} \text {. }
$$

Among many test problems, the following four examples are considered

$$
\begin{aligned}
& f_{1}(x)=x^{2}-9, \quad x^{*}=3, x_{0}=2.6 \\
& f_{2}(x)=(x-2)\left(x^{6}+x^{3}+1\right) e^{-x^{2}}, \quad x^{*}=2, x_{0}=1.8, \\
& f_{3}(x)=\prod_{k=1}^{12}(x-k), \quad x^{*}=5, x_{0}=5.3 .
\end{aligned}
$$


To sum up, it can be concluded the that the method (1.1) has fifth-order convergence. Therefore, authors' claim is not true that they have presented a family of iterative methods for solving nonlinear equations with eighth-order convergence.

Acknowledgments The author thank to the anonymous referees for their valuable comments and for the suggestions to improve the readability of the paper.

Open Access This article is distributed under the terms of the Creative Commons Attribution License which permits any use, distribution, and reproduction in any medium, provided the original author(s) and the source are credited.

\section{Reference}

1. Khan, Y., Fardi, M., Sayevand, K.: A new general eighth-order family of iterative methods for solving nonlinear equations. Appl. Math. Lett. 25, 2262-2266 (2012) 